第 Springer

Kew/

PLANTS PEOPLE

POSSIBILITIES

\title{
Some New Species from Burma
}

Author(s): J. H. Lace

Source: Bulletin of Miscellaneous Information (Royal Botanic Gardens, Kew), Vol. 1915, No. 9 (1915), pp. 393-407

Published by: Springer on behalf of Royal Botanic Gardens, Kew

Stable URL: http://www.jstor.org/stable/4113228

Accessed: 27-06-2016 02:47 UTC

Your use of the JSTOR archive indicates your acceptance of the Terms \& Conditions of Use, available at

http://about.jstor.org/terms

JSTOR is a not-for-profit service that helps scholars, researchers, and students discover, use, and build upon a wide range of content in a trusted digital archive. We use information technology and tools to increase productivity and facilitate new forms of scholarship. For more information about JSTOR, please contact support@jstor.org.

Springer, Royal Botanic Gardens, Kew are collaborating with JSTOR to digitize, preserve and extend access to Bulletin of Miscellaneous Information (Royal Botanic Gardens, Kew) 
ROYAL BOTANIC GARDENS, KEW.

\author{
B U L L E T I N
}

OF

\title{
MISCELLANEOUS INFORMATION.
}

No. 9]

\section{XLI.-SOME NEW SPECIES FROM BURMA.}

\section{J. H. LACE.}

During the last five years a considerable number of new species of plants from Burma have been described and published in the Kew Bulletin or in the Records of the Botanical Survey of India, the majority of which were found in Maymyo and its neighbour. hood or in the Southern Shan States, whilst others came chiefly from the hill regions of the Ruby Mines and Bhamo Districts. Although shrubs, climbers and herbaceous plants predominate amongst the new species, many trees, some of great size, are also included, for instance, Manglietia Hookeri, Cubitt \& Smith, Elaeocarpus Robertsonii, Gamble, Paranephelium hystrix, W. W. Smith, Meliosma Mannii, Lace, Dalbergia maymyensis, Craib, Acacia Myaingii, Lace, Pyrus kachinensis, W. W. Smith, Lagerstroemia Collettii, Craib, Homalium bhamoense, Cubitt \& Smith, Adina indivisa, Lace, Diospyros glandulosa, Lace, Styrax Lacei, W. W. Smith, Stereospermum grandiflorum, Cubitt \& Smith, Beilschmedia Robertsonii, Gamble, all from Upper Burma, while from Lower Burma may be mentioned Rhus amherstensis, W. W. Smith, Millettia utilis, Dunn, Cordia globifera, W. W. Smith and Wightia Lacei, Craib. In the same period other additions have been made to the flora of Burma by the collection of species previously recorded only from neighbouring countries, e.g., Miliusa Thorelii, Finet \& Gagnep., Sterculia Principis, Gagnep., Chailletia gelonioides, Hook. f., Celastrus Hookeri, Prain, Acer oblongum, Wall., Meliosma Thomsonii, King ex Brandis, Dracontomelum mangiferum, Bl., Mucuna sempervirens, Hemsl., Pueraria alopecuroides, Craib, Dalbergia Kerrii, Craib, Saraca cauliflora, Baker, Rubus lucens, Focke, Rubus yunanicus, Kuntze, Rosa sericea, Lindl., Distylium indicum, Benth., Viburnum erubescens, Wall., Viburnum inopinatum, Craib, Hymenodictyon flaccidum, Wall., Gardenia sootepensis, Hutch., Rhododendron indicum, Sweet, R. microphytum, Franch., R. oxyphyllum, Franch., Styrax grandiflorum, Griff., S. Veitchiorum, Franch., Fraxinus Griffithii, Clarke, Strychnos Thorelii, Pierre, Endiandra frma, Nees, Cleistanthus chartaceus, Muell.-Arg., Glochidion Kerrii, Craib, Sauropus

(4108.) Wt. 153-601. 1,125. 11/15. J. T. \& S. G. 14. 
bicolor, Craib, Antidesma sootepensis, Craib, Macaranga triloba, Muell.-Arg., Macaranga indica, Wight, Quercus Junghuhnii, Miq., Quercus Rex, Hemsl.

The above tends to show that our knowledge of the flora of Burma is by no means complete, even in the case of trees; and, taking into consideration the fact that most of the recent collectors were officers of the Forest Department who naturally pay most attention to woody species, often to the entire exclusion of herbaceous plants without economic value, it appears safe to infer that there is still much to be done in the botanical exploration of the country, especially in Upper Burma and more particularly in those regions whose elevation exceeds $3000 \mathrm{ft}$. above sea level.

Amongst the species described below, the majority of which were found at Maymyo, perhaps the most interesting is Allospondias laxiflora, a large deciduous tree on the small limestone hills near the lower reaches of the Salween river and its tributaries, about which doubt has existed since flowering specimens of it were first collected by Brandis in 1862 ; this was originally named Buchanania laxiflora, Kurz, but M. Pierre pointed out that it was not a Buchanania and was probably a new genus, a fact admitted in Indian Trees, p. 206 ; there is no leaf on the sheet of the specimen collected by Brandis which is in the Kew Herbarium, and it is not known what leaf (simple) was described by Kurz under Buchanania laxiflora in his Flora of British Burma.

With regard to the local flora of Maymyo, of which I was able to make a fairly complete collection, any general description would at present be out of place and is reserved for some future occasion. I gladly take this opportunity, however, of acknowledging with many thanks the kindness of Sir David Prain, Director of the Royal Botanic Gardens, in permitting the work of naming my collections from Burma to be done in the Kew Herbarium, and the assistance afforded me by members of the Staff. To Mr. W. G. Craib, lately Assistant for India in the Herbarium, I am especially indebted both for the keen interest he has taken in my collections and for his valuable help and sound criticisms.

Clematis burmanica, Lace [Ranunculaceae-Clematideae]; a C. Craibiana, Lace, cui valde affinis, nervatione, indumento, filamentis multo longioribus, antheris conspicue minoribus, stigmato clavato inter alia differt.

Frutex scandens; rami graciles, teretes, sulcati, purpureobrunnei, pruinosi, juventute pilis brevibus pallide luteis nitidis adpressis puberuli. Folia pinnatim 3-foliata; petiolus $8-10 \mathrm{~cm}$. longus, parum costatus ut ramuli puberulus; foliola late ovata, apicem acutum versus gradatim angustata, basi rotundata, plerumque anguste cordata, interdum truncata vel ramulorum apices versus cuneata, usque ad $10.5 \mathrm{~cm}$. longa et $5.5 \mathrm{~cm}$. lata, terminali quam lateralibus parum majore, chartacea, juventute pilis brevibus pallide luteis adpressis praecipue ad nervos sparse tecta, mox pagina utraque fere glabra, inferiore pallidiore glaucescente, e basi vel fere e basi 7 -nervia, nervis gracilibus supra magis minusve impressis subtus prominentibus, nervulis fuscobrunneis reticulationem gracilem formantibus, margine integra; 
petioluli usque ad $3 \mathrm{~cm}$. , plerumque circa $1.5 \mathrm{~cm}$. longi. Flores in paniculas subangustas paucifloras axillares et terminales plerumque foliis duobus unifoliolatis instructas dispositi; paniculae ad $30 \mathrm{~cm}$. longae, pedunculo communi ad $13 \mathrm{~cm}$. longo suffultae; pedunculi partiales ad $5 \mathrm{~cm}$. longi, bracteae variabiles, saepius 5-7 mm. longae. integrae, saepe amplexicaules, pubescentes ; pedicelli 1-3 cm.longi, ut pedunculi indumento glanduloso pallide Iuteo plus minusve deciduo tecti. Sepala alba, patula, ad $1.5 \mathrm{~cm}$. longa et $0.6 \mathrm{~cm}$. lata, multinervia, apice mucronata, parum reflexa, extra praesertim ad basem et margines prope pubescentia, intra glabra. Filamenta $6 \mathrm{~mm}$. longa, ligulata, glabra; antherae $1.5 \mathrm{~mm}$. longae, apice rotundatae. Ovarium pallide brunneohirsutum; stylus circa $4 \mathrm{~mm}$. longus, plumosus, stigmate clavato.

Indo-China. Burma: near Maymyo, Ani Sakan, 900 m., Lace 5927.

Clematis Craibiana, Lace [Ranunculaceae-Clematideae]; a C. Meyeniana, Walp. paniculis multo longioribus, foliolis tenuioribus basi rarissime leviter cordatis distinguenda.

Frutex scandens, ramulis gracilibus teretibus sulcatis pallidis vel purpureo-brunneis pilis albis brevibus mollibus sparse tectis. Folia pinnatim trifoliata, petiolo communi 4-7 $\mathrm{cm}$. longo indumento ut ramulis tecto suffulta ; foliola ovato-lanceolata vel ovata, apice acuminata, obtusiuscula, basi rotundata, rotundato-cuneata, truncata vel inferiora interdum leviter cordata, usque ad $11 \mathrm{~cm}$. longa et $7 \mathrm{~cm}$. lata, chartacea, glabra, subtus pallidiora, e basi vel fere e basi 5-nervia, nervis in foliis maturis pagina utraque sed praesertim inferiore conspicuis, reticulata, integra, petiolulis 2-5 cm. longis suffulta. Paniculae axillares, laxae, pluriflorae, ad $42 \mathrm{~cm}$. longae, pedunculo communi ad $15 \mathrm{~cm}$. longo suffultae; pedunculi partiales ad $5 \mathrm{~cm}$. longi, ut pedunculus communis sulcati indumentoque ut ramuli praesertim ad nodos tecti; bracteae apice bi-vel tri-dentatae, interdum foliaceae, ad $7 \mathrm{~mm}$. longae; pedicelli $1.5-4 \mathrm{~cm}$. longi. Sepala $1.5 \mathrm{~cm}$. longa, 3-5 mm. lata, alba, brunneo-nervosa, extra pallide luteo-puberula, intus glabra, apice truncato saepe reflexo. Filamenta $3-3.5 \mathrm{~mm}$. longa, complanata, glabra, antheris $3.5 \mathrm{~mm}$. longis connectivo excurrente breviter apiculatis. Ovarium dense albo-hirsutum; stylus plumosus, circiter $5.5 \mathrm{~mm}$. longus, stigmate haud incrassato.

Indo-China. Burma: Maymyo Plateau, on shrubs and trees near stream, 1100 m., Lace 6122. Named after Mr. W. G. Craib who has assisted so much in working out plants from Maymyo.

Polygala pellucida, Lace [Polygalaceae]; a P. glaucescente, Wall., foliis crassioribus, floribus majoribus, sepalo uno persistente recedit.

Herba erecta, ad $20 \mathrm{~cm}$. alta, caule angulato glabro parce divaricatim ramoso. Folia oblongo-oblanceolata, acuta, apiculata, basi in petiolum angustata, usque ad $3.5 \mathrm{~cm}$. longa et $1.3 \mathrm{~cm}$. lata, coriacea, glabra, pagina superiore sicco rugosa. inferiore pallidiora; costa supra impressa subtus prominente, nervis lateralibus utrinsecus 6-8 inconspicuis vel subtus fere pro. minulis, margine recurva, petiolo $1-3 \mathrm{~mm}$. longa suffulta. Racemi usque ad $7 \mathrm{~cm}$. (pedunculo communi $0.5-2 \mathrm{~cm}$. longo 
incluso) longi; pedicelli $0 \cdot 5-1 \mathrm{~mm}$. longi; bracteae minutae, cito deciduae; flores sulphurei, $5 \mathrm{~mm}$. longi. Sepala tria exteriora inter se subaequalia, ovata vel oblongo-ovata, apice rotundata, $2 \mathrm{~mm}$. longa, aureo-pellucido-punctata, mediano persistente, duo interiora aliformia, apice cucullata, basi cuneata, $4 \mathrm{~mm}$. longa. Petala lateralia oblonga, apice rotundata, $3.5 \mathrm{~mm}$. longa; carina cucullata, non cristata, $4 \mathrm{~mm}$. longa. Stigma truncatum. Capsula obcordata, 3-3.5 mm. lata, margine superne in alam gradatim expansa, nervis satis conspicuis fere e capsulae apice radiantibus; semina globosà, parum lateraliter compressa, atra, minute tuberculata, pilis brevibus albis sparse tecta.

Indo-China. Burma: Maymyo Plateau, in marshy grasslands and on banks of streams, $1050 \mathrm{~m}$., Lace 4844.

Buettneria integrifolia, Lace [Sterculiaceae-Buettnerieae]; ab affini $B$. erosa, Gagnep, foliis integris facile distinguenda.

Frutex grandis, scandens, ramulis inarmatis prominenter pluricostatis brunneis juventute pilis minutis stellatis hic illic instructis. Folia late ovata vel oblongo-ovata, apics subito longe acuminata, truncata, mucronata, basi cordata, lobis rotundatis, usque ad $20 \mathrm{~cm}$. longa et $14.5 \mathrm{~cm}$. lata, sicco tenuia, glabrescentia, praecipue ad nervospilis paucis minutis stellatis hic illic instructa, e basi 5-nervia, nervis lateralibus (e costa ortis) utrinsecus 5-6 arcuatis intra marginem anastomosantibus cum nervis primariis pagina utraque prominentibus, nervis tranversis numerosis inter se parallelis pagina utraque conspicuis, margine integra, parum undulata, petiolo 3-11 cm. longo supra canaliculato sparse stellatopubescente praecipue apicem versus suffulta; stipulae lanceolatae vel ovato-lanceolatae, acuminatae. Inflorescentia generis, sparse minuteque stellato-pubescens; pedunculi sulcati; pedicelli gracillimi, 4-7 mm. longi, medium versus articulati ; alabastra conoidea, angulata; flores 0.8-1 cm. diametro. Sepala lanceolata, conspicue nervosa, acuta, 4-5 mm. longa, 1-1.5 mm. lata, basi inter se connata, minute sparse puberula, pellucido-notata. Petala ungui $2 \mathrm{~mm}$. longo suffulta, limbi lobis deltoideis acutis $2-2.5 \mathrm{~mm}$. latis, apice truncata, ad staminum tubum affixa, appendice anguste lanceolata 3-3.5 $\mathrm{mm}$. longa glabra. Ovarium parum pubescens.

Indo-China. Burma: near Maymyo Plateau, Gokteik Gorge, $550 \mathrm{~m}$. , Lace 5454.

China. Yunnan: Szemao, 1530 m., Henry 13,370.

Euonymus longipes, Lace [Celastraceae-Celastreae]; ab $E$. acanthocarpo, Franchet, foliis saepe integris majoribus, pedunculo longiore robustiore, ab $E$. cinereo, Lawson, foliis saepe integris, inflorescentia multo longiore recedit.

Frutex grandis vel arbuscula, omnino glabra; ramuli quadranguli, bifacialiter canaliculati; rami teretes, tuberculis minutis dense tecti. Folia oblongo-obovata ad ovata, apice obtuse acuminata, basi cuneata rotundatave, interdum parum inaequilateralia, $6-11.5 \mathrm{~cm}$. longa, $3-5.5 \mathrm{~cm}$. lata, coriacea, juvenilia viridia, matura griseo-viridia, subtus parum pallidiora, nervis lateralibus utrinsecus 5-6 obliquis intra marginem anastomtsantibus pagina utraque conspicuis, margine parte dimidia superiore saepe 
obscurius crenulata, rarius crenata; petioli $1 \mathrm{~cm}$. longi, supra conspicue canaliculati. Cymae axillares, pedunculo communi $4-9.5 \mathrm{~cm}$. longo ut ramulis quadrangulari et bifacialiter canaliculato suffultae; pedunculi partiales $1-3.5 \mathrm{~cm}$. longi, ultimi pedicellis subaequilongi; bracteae minutae, triangulares, serrulatae, fugases; pedicelli graciles, saepius $4 \mathrm{~mm}$. longi; flores 4-meri. Calycis lobi, rotundati, recurvi, $2 \mathrm{~mm}$. lati. Petala viva viridi-alba, orbicularia $3 \mathrm{~mm}$. diametro, late unguiculata. Filamenta $1.5 \mathrm{~mm}$. longa, antheris globosis. Discus $2.5 \mathrm{~mm}$. diametro. Ovarium echinatum, stylo paulo ultra $1 \mathrm{~mm}$. longo.

Indo-China. Burma: Maymyo Plateau, an undershrub or small tree in damp localities, $1050 \mathrm{~m}$., Lace 6149.

Allospondias laxiflora, Lace [Anacardiaceae-Spondieae]; ab A. lakonensi, Stapf, foliis multo majoribus, panicula terminali, fructu eum Odinae simulante recedit.

Arbor excelsa, decidua, cortice laevi argenteo. Folia alterna, apices versus ramulorum sita, imparipinnata, usque ad $65 \mathrm{~cm}$. petiolo communi $5-10 \mathrm{~cm}$. longo incluso longa; foliola opposita vel subopposita interdumve alterna, utrinque 6-9 juga, ovatolanceolata, ovato-oblonga oblongave apice abruptius longe acuminata, acuta, basi saepius rotundata, interdum inaequilateralia, superiora interdum latere altero cuneata, altero rotundata, usque ad $1 \% \mathrm{~cm}$. longa et $6.5 \mathrm{~cm}$. lata, inferioribus quam aliis conspicue minoribus, chartacea, pilis albis divaricatis pagina superiore hic et illic instructa, inferiore ad nervos nervulosque densius instructa, subtus pallidiora, nervis lateralibus utrinque 12-14 brunneis arcuatis subtus prominulis, margine integra, sessilia, terminali a lateralibus $1-2.5 \mathrm{~cm}$. distante quam aliis parum minore, basi cuneata; rhachis ut petiolus teres sed sicco plus minusve canaliculata pilis brunneis brevibus molliter tomentosa. Flores ante folios evoluti, in paniculam copiose ramosam terminalem pubescentem $50 \mathrm{~cm}$. vel ultra longam dispositi; bracteae subulatae linearesve, 1-1.5 mm. longae; pedicelli brevissimi, aggregati. Sepala 5, libera, imbricata, ovata, apice obtusa, circiter $0.5 \mathrm{~mm}$. longa, dorso puberula, persistentia sed haud accrescentia. Petala 5 , viva alba, ovata, apice parum cucullata, $2.5-3 \mathrm{~mm}$. longa, 3-nervia, dorso minute puberula. Stamina 10, filamentis inferne dilatatis circa $1 \mathrm{~mm}$. longis minutissime puberula. Discus vivus aurantiacus, 5-lobatus, lobis retusis. Styli 5, liberi, stigmatibus truncatis. Drupa oblonga, basi inaequilateralia, circa $1 \cdot 2 \mathrm{~cm}$. longa; semina solitaria, pendula, radicula superiore.-Buchanania laxiflora, Kurz in Journ. As. Soc. Beng., vol. xli. p. 304 et For. Fl. Burma, vol. i. p. 30 ; Brandis, Indian Trees, p. 206-certe quoad flores, foliorum descriptione exclusa.

Burma. Thaton District: a conspicuous tree on the dry limestone hills which rise suddenly out of the plain on both banks of the lower Salween river, Lace 4574 (flowers), J. C. Murray (fruit and leaves).

Burmese name, Taung-gwe.

Uraria barbata, Lace [Leguminosae-Hedysareae]; ab $U$ : cordifolia, Wall., cui affinis, foliis tenuioribus conspicue ciliatis, fructu pilis apice uncinatis tecto differt. 
Suffrutex grandis, ramis crassis pallide brunneis striatis pilis longis albis divaricatis tectis. Folia late ovata, acuminata, apiculata, basi anguste cordata vel rarius truncata, usque ad $26 \mathrm{~cm}$. longa et $22 \mathrm{~cm}$. lata, chartaceo-membranacea, pagina utraque pilis longis divaricatis basi tuberculatis parce instructa, nervis lateralibus utrinsecus 10-11, nervis transversis inter se parallelis, margine conspicue dense ciliata; petiolus $1-3 \mathrm{~cm}$. longus, indumento ei ramorum simili tectus; stipulae scariosae, striatae, dorso pilis longis albis instructae, diu persistentes, Inflorescentia e racemis longis et axillaribus et terminalibus laxe paniculatis constituta, usque ad $80 \mathrm{~cm}$. longa ; flores 3-12-fasciculati; bracteae scariosae, lineari-lanceolatae, longe acuminatae, valde nervosae et ciliatae; pedicelli $5-7 \mathrm{~mm}$. longi, glandulosopubescentes. Calycis ante fructum maturum decidui tubus circa $1.5 \mathrm{~mm}$. longus, lobi longe subulato-acuminati, infimo ad $3.5 \mathrm{~mm}$. longo; calyx totus sed praesertim lobi pilis longis albis divaricatis tectus. Vexillum obovatum, apice rotundatum, basi cuneatum, haud auriculatum, $5 \mathrm{~mm}$. longum; alae et carina vexillo breviores. Staminis vexillaris filamentum $3.5 \mathrm{~mm}$. longum. Legumen pilis apice uncinatis tectum; semina 1-6, brunnea, laevia, haud nitida.-U. cordata, Wall., var. barbata, Wall. Cat. 5679 B et C.

Indo-Cirna. Burma: Katha District, Lace 4811; Ruby Mines District, Wapyudaung, 370 m., Lace 5981 ; near Maymyo Plateau, Gokteik Gorge, 615 m., Lace; Upper Chindwin, Tamu, Meebold 7597; T'aong Dong, Wall. Cat. 5679 B. Cult. Hort. Bot. Calc. e Taong Dong, Wall. Cat. 5679 C.

Mucuna Collettii, Lace [Leguminosae-Phaseolae]; a M. macrocarpa, Wall., foliolorum forma et indumento facile distinguenda.

Frutex late volubilis, caule basi $0.5 \mathrm{~m}$. diametro, ramis crassis, cortice laevi, ramulis fulvo-pubescentibus. Stipulae triangulares, acuminatae, acutae, $3-4 \mathrm{~mm}$. longae, cito caducae; petiolus $6-15.5$ $\mathrm{cm}$. longus, striatus, tomento rubiginoso deciduo tectus; foliola lateralia inaeqilateralia, latere altero dimidiatim lanceolatooblonga, basi late cuneata, altero dimidiatim late ovata, basi anguste cordata, apice saepius breviter acuminata sed interdum rotundata et emarginata, 11-15 cm. longa, 6-10 cm. lata; foliolum terminale a lateralibus $3 \cdot 5-4 \mathrm{~cm}$. distans, ellipticum vel oblongoellipticum, usque ad $17 \mathrm{~cm}$. longum et $10 \mathrm{~cm}$. latum; foliola omnia apiculata, chartacea, viridia, supra primo sparse setosa, demum glabra, subtus molliter cupreo-brunneo-tomentosa, nervis lateralibus utrinsecus 5-7 supra conspicuis subtus prominentibus, petiolulis validis $0.7-1 \mathrm{~cm}$. longis suffulta. Inflorescentia racemiformis, e ligno vetustiore fasciculatim orta, usque ad $20 \mathrm{~cm}$. longa, rhachi brunneo-pubescente; pedicelli validi, recti curvative, ad $1.5 \mathrm{~cm}$. longi, solitarii vel $2-3$ pedunculis brevissimis secondariis gesti, molliter pubescentes. Calyx diu persistens, extra pilis brevibus griseo-brunneis et setis satis numerosis pallide luteobrunneis tectus, intra brunneo-tomentosus, tubo $1.5 \mathrm{~cm}$. diametro circiter $1 \mathrm{~cm}$. longo saepe obliquo, lobis supremis quam infimo 7-9 $\mathrm{mm}$. longo multo brevioribus et latioribus, lateralibus $5 \mathrm{~mm}$. longis. Corolla 5-6 cm. longa, purpurea vel purpureo-viridis; vexillum late ellipticum, $2.8 \mathrm{~cm}$. diametro, carina circiter dimidio brevior, superne ciliolatum; alae extra basem versus pubescentes, 
superne et inferne ciliatae, medio non ciliatae; carina alas superans, basi ciliata. Staminum tubus $5 \mathrm{~cm}$. longus, antheris filamentorum brevium barbatis. Ovarium pilis rigidis brevibus obtectum, stylo barbato superne glabro. Legumina ad $38 \mathrm{~cm}$. longa, $3.5-4 \mathrm{~cm}$. lata, $1-1.5 \mathrm{~cm}$. crassa, inter semina constricta, valvis planis, suturis obliquis, apice breviter acuminata, basi cuneata, stipite brevissimo suffulta, tomento rufo tecta demum glabra nisi ad suturas. Semina planiuscula, oblonga orbiculariave, ad $2 \cdot 8 \mathrm{~cm}$. longa et $2 \cdot 3 \mathrm{~cm}$. lata, fusco-brunnea vel nigra, hilo pallido margine tota quarto breviore.

Indo-China. Burma: Maymyo Plateau, 1050 m., Lace 5866; C. G. Rogers 19; Hauxwell; Shan Hills, 1230 m., Collett 458.

China. Yunnan: Szemao, 1530 m., Henry 11,702.

Pueraria Lacei, Craib [Leguminosae-Phaseoleae]; a P. alopecuroide, Craib, floribus multo majoribus facile distinguenda.

Suffrutex late scandens; ramuli $4 \mathrm{~mm}$. diametro, ferrugineohirsuti. Folia pinnatim trifoliolata, petiolo $4-7.5 \mathrm{~cm}$. longo suffulta; stipulae oblongae, apice rotundatae, basi bilobae, medio affixae, $2 \mathrm{~cm}$. longae, $7 \mathrm{~mm}$. latae; foliola ovata vel terminalia subrhomboidea, apice acuminata sensim acutata, basi lateralia rotundata, terminalia obtuse cuneata, 6-9 cm. longa, $3 \cdot 3-5 \cdot 5 \mathrm{~cm}$. lata, subchartacea, margine integra, ciliata, supra tenuiter adpresse pilosa, subtus sericea, nervis lateralibus utrinque circiter 6 supra conspicuis subtus prominentibus, nervis transversis obscuris; petioluli $0.5 \mathrm{~cm}$. longi, foliolo terminali a lateralibus $2-2.5 \mathrm{~cm}$. distante; stipellae lineares, acutae, $1 \cdot 2-1.5 \mathrm{~cm}$. longae, usque ad $1.5 \mathrm{~mm}$. latae. Racemi solitarii axillares vel ad apices ramulorum paniculati, pedunculo communi 7-10 cm. longo bracteis aliquot vacuis stipulis forma similibus sed minoribus instructo; bracteae lineari-lanceolatae 1-1.5 $\mathrm{cm}$. longae, 2-3 $\mathrm{mm}$. latae, ferrugineo-pilosae; pedicelli $3 \mathrm{~mm}$. longi, apice bracteolis 2 ovato-lanceolatis acutis $4 \mathrm{~mm}$. longis $1.5 \mathrm{~mm}$. latis instructi. Calycis extus ferrugineo-hirsuti intus appresse hirsuti, tubus circiter $0.5 \mathrm{~cm}$. longus, lobi duo supremi in unum paulo ultra $1 \mathrm{~cm}$. longum, apice breviter bifidum connati, lobus infimus lateralibus paulo longior, $1.5 \mathrm{~cm}$. longus. Corolla longe exserta; vexillum reflexum late ellipticum, emarginatum, basi auriculatum, $1.4 \mathrm{~cm}$. longum, $1 \cdot 2 \mathrm{~cm}$. latum, ungui $4 \mathrm{~mm}$. longo suffultum; alae oblongae, basi longe appendiculatae, $1.3 \mathrm{~cm}$. longae, ungui $0.5 \mathrm{~cm}$. longo; carina obtusa inappendiculata, $1.1 \mathrm{~cm}$. longa, $0.5 \mathrm{~cm}$. lata, ungui $6 \mathrm{~mm}$. longo. Stamen vexillare ima basi liberum, medio cum ceteris connatum; antherae uniformes, parvae. Ovarium stipitatum, adpresse hirsutum. stylo brevi glabro, stigmate parvo capitato pilis perpaucis instructo.

Indo-China. Burma: Thayetmyo District, Lace 2685.

Eriosema pilosum, Lace [Leguminosae-Phaseoleae]; ab $E$. chinense, Vogel, indumento longo albo facile distinguendum.

Herba perennis; caules graciles, $12-21 \mathrm{~cm}$. alti, lignosi, striati, simplices furcative. Folia lanceolata, oblongo-lanceolata vel oblonga, minute apiculata, basi rotundata, ad $3.8 \mathrm{~cm}$. longa, $1 \mathrm{~cm}$. 
lata, chartaceo-membranacea, supra viridia, pilis longis albidis divaricatis praetereaque brevius pubescentia, subtus griseo-albida, tomento denso albido et glandulis aureis et ad nervos pilis longis albis divaricatis instructa, nervis lateralibus utrinque 6 supra impressis subtus prominulis, margine parum incurva, longe ciliata, petiolo 1-2 $\mathrm{mm}$. longo indumento ut caule instructo suffulta; stipulae scariosae, angustae, longe acuminatae, 3-4 mm. longae, valde nervosae, persistentes. Flores axillares, subsessiles, lutei. Calyx $5 \mathrm{~mm}$. longus, dentibus acutis, infimo aliis parum longiore, extra glanduloso-pilosus, intra ad dentes pilis paucis albis adpressis instructus. Vexillum 8-9 $\mathrm{mm}$. longum, rotundatum, apice emarginatum, basi cuneatum, auriculatum, dorso pilosum; alae $\tau-8 \mathrm{~mm}$. longae, fere glabrae; carina $6 \mathrm{~mm}$. longa, apice rotundata, dorso glanduloso-pilosa. Ovarium pilis longis albis obtectum, stylo glabro, stigmate parvo capitato. Legumen oblongum, apiculatum, 0.8-1 cm. longum, 0.5-0.6 cm. latum, glandulosum, pilis longis albis dense pilosum; semina oblonga, brunnea, fusco-maculata, glabra, $3 \mathrm{~mm}$. longa.

Indo-China. Burma: Maymyo Plateau, 1050 m., Lace 6328.

Bauhinia sericea, Lace [Leguminosae-Bauhinieae]; B. glabrifoliae, Baker et $\boldsymbol{B}$. piperifoliae, Roxb., affinis, ab illa floribus majoribus, ab hac ovarii suturis villosis recedit.

Frutex late scandens, cirrhosus; ramuli primo griseo-tomentelli, cito glabri, demum brunnei, sulcati. Folia late vel anguste ovata, saepius plus minusve bifida, saepe in ramulis juvenilibus usque ad basem, saepe prope inflorescentiam integra et apice rotundata, lobis plus minusve triangularibus apice obtusis, basi truncata vel late haud alte cordata, usque ad $13.5 \mathrm{~cm}$. longa et 11.5 $\mathrm{cm}$. lata, subcoriacea, nitida, glabra nisi nervis basi pilis paucis primo instructis, e basi 7 -nervata, duobus basalibus minus conspicuis saepe additis, nervis supra conspicuis subtus prominentibus, nervulis rete laxum pagina utraque conspicuum formantibus, petioli ad $6 \mathrm{~cm}$. longi, utrinque tumidi, primo breviter arcte albopubescentes, mox glabri. Flores in paniculas corymbiformes 8-13 cm. diametro dispositi; alabastra globosa, breviter acuminata, subsericea; bracteae angustae, acutae, $4 \mathrm{~mm}$. longae; pedicelli $1 \cdot 5-2 \mathrm{~cm}$. longi, graciles, breviter sericei; bracteolae duae, angustissimae, circiter $2 \mathrm{~mm}$. longae, bene infra pedicelli medium sitae. Calycis tubus $3-3.5 \mathrm{~mm}$. longus, limbo saepius 3-fido 5-6 $\mathrm{mm}$. longo intra glabro. Petala viva gilvo-albida, obovata, unguiculata, $0 \cdot 8-1 \mathrm{~cm}$. longa, tribus superioribus margine undulatis, omnia extra medio dense pubescenta et posteriore fere glabro excepto intus ad medium et ad unguem pubescentia. Stamina fertilia 3 , filamentis $1 \cdot 2-1 \cdot 4 \mathrm{~cm}$. longis glabris superne angustatis, antheris oblongis $2.5 \mathrm{~mm}$. longis ; staminodia 3, filamentis gracillimis $5 \mathrm{~mm}$. longis. Ovarium ad suturas villosum. lateribus glabrum, stipite brevi; stylus primo incurvus, ventrice fere ad apicem villosus; stigma mediocre.

Indo-China. Burma: Ani Sakan and Maymyo, 800-1050 m., Lace 6208.

Ripe pods, believed to belong to this species, were found on the ground in the hot weather. They are very dark brown in colour, 
shining, glabrous, slightly curved, $37-40 \mathrm{~cm}$. long, $3 \cdot 5-4 \cdot 2 \mathrm{~cm}$. broad and about $1.3 \mathrm{~cm}$. thick at the seeds, the apex is rounded and apiculate and the base gradually narrowed into the $6-10 \mathrm{~cm}$. long stipe. The valves are strongly nerved, considerably constricted between the seeds and thickened along both sutures. Seeds 14-18, narrowly obovate or elliptic, turgid, dark brown, about $2.2 \mathrm{~cm}$. long by $1.4 \mathrm{~cm}$. across.

Acacia insuavis, Lace [Leguminosae-Mimoseae]; ab A. pinnata, Willd., foliis longioribus, foliolis majoribus, capitulis majoribus longius pedunculatis, inter alia recedit.

Frutex grandis, scandens, ramis late extensis cinereo-corticatis, ramulis juventute viridibus striatis molliter crispatim albo-pubescentibus lenticellatis; ramuli, petioli inflorescentiaeque ramuli aculeis rectis $1 \mathrm{~mm}$. longis vel in ramulis usque $3 \mathrm{~mm}$. attingentibus longioribus interdum parum curvatis pallide brunneis glabris hic illic instructi vel interdum inarmati. Folia $16-25 \mathrm{~cm}$. longa (petiolo excluso), petiolo $3-5 \mathrm{~cm}$. longo pubescente striato prope basem glandula oblonga sessili ornato suffulta, rhachi dense crispatim tomentosa superne glandulis 1-4 parvis sessilibus instructa; stipulae parvae, triangulares, cito caducae; pinnae utrinque $13-30,2 \cdot 5-9.5 \mathrm{~cm}$. longae, rhachi dense crispatim albotomentosa ; foliola utrinque 30-80, imbricata, anguste linearia, mucronata, basi inaequilateralia, truncata, $5 \mathrm{~mm}$. longa, vix $1 \mathrm{~mm}$. lata, nervulis obscuris, pagina utraque glabra, ciliolata. Capitula in paniculas magnas terminales foliatas disposita, pallide iutea, $1-1 \cdot 2 \mathrm{~cm}$. diametro, pedunculis $2-5 \mathrm{~cm}$. longis solitariis vel usque ad 5 in axillo quoque pilis brevibus crispatis albis instructis suffulta; bracteae spatulatae, pubescentes, $1.5 \mathrm{~mm}$. longae. Caly $x$ infundibuliformis, $2.5 \mathrm{~mm}$. longus, extra puberulus, lobis triangularibus acutis vix $1 \mathrm{~mm}$. longis ciliatis. Corolla $3 \mathrm{~mm}$. lorga, lobis triangularibus acutis $1 \mathrm{~mm}$. longis ciliatıs dorso pubescentibus. Stamina numerosissima (ultra 100), 4.5-5 mm. longa, basi parum connata, glabra, antheris parvis apice glandula stipitata fugace ornatis. Ovarium sericeum, stipite $1.5 \mathrm{~mm}$. longo suffultum, stylo $4 \mathrm{~mm}$. longo stamina superante.

Indo-China. Burma: Ani Sakan, near Maymyo, 900 m., Lace 61i3. Burmese name, Subok.

Occurs in many parts of Burma, in forests and near villages where it is probably sometimes cultivated. The branshes and leaves when crushed give out a most foetid odour.

Acacia macrocephala, Lace [Leguminosae-Mimoseae]; ab A pennata, Willd., capitulis multo majoribus facile distinguenda. Frutex scandens; ramuli brunnei, striati, multilenticellati, primo ut petioli, rhachis pedunculique tomento brevi denso rubiginoso furfuraceo-glanduloso tecti, aculeis parvis curvatis. Folia usque ad $22 \mathrm{~cm}$. longa, petiolo circa $4 \mathrm{~cm}$. longo striato glandula oblonga sessilia $0.75 \mathrm{~mm}$. alta basin versus instructo suffulta; rhachis canaliculata superne glandulis tribus sessilibus instructa; stipulae circa $3 \mathrm{~mm}$. longae, cito deciduae; pinnae utrinque 12-13, rhachi crispatim pubescente, $3-8.5 \mathrm{~cm}$. longae, foliola utrinque 35-65, inferiora parum imbricata, linearia, acuta, basi truncata, $5-8 \mathrm{~mm}$. longa, $1 \mathrm{~mm}$. lata, costa tenui supra fere obsoleta infra 
inconspicua, nervis saepissime omnino obscuris, glabra, praesertim inferne sparse ciliata. Capitula in paniculas terminales foliatas disposita, 2-2.5 cm. diametro, pedunculis $3.5-4.5 \mathrm{~cm}$. solitariis vel usque 5-fasciculatis suffulta; bracteae ultra $2 \mathrm{~mm}$. longae, lineares, apice latiores et incrassatae, glanduloso-pubescentes. Calyx infundibuliformis, $3 \cdot 5-4 \mathrm{~mm}$. longus, extra pubescens, intra glaber, lobis $1.5-2 \mathrm{~mm}$. longis deltoideis lateribus parum convexis, acutis. Corolla $5 \mathrm{~mm}$. longa, nervo mediano brunneo conspicuo, lobis $1.5 \mathrm{~mm}$. longis deltoideis lateribus parum convexis obtusiusculis vel sicco ob marginem incurvum breviter pseudoacuminatis ciliolatis dorso breviter puberulis. Stamina libera, numerosissima, filamentis $1 \mathrm{~cm}$. longis glabris, antheris apice glandula stipitata decidua ornatis. Ovarium breviter hirsutum, stipite $1.5 \mathrm{~mm}$. longo glabro suffultum, stylo $0.8 \mathrm{~cm}$. longo inferne sparse puberulo.

Indo-China. Burma : Bhamo District, Sinlum Kaba, 1700 m., Lace 5787.

Albizzia crassiramea, Lace [Leguminosae-Mimoseae]; ab A. Gamblei, Prain, cui affinis, foliolis majoribus, legumine rubrobrunnea haud stramineo ad semina prominenter laxe reticulato distinguenda.

Arbor mediocris; ramuli validi, $8 \mathrm{~mm}$. diametro, striati, puberuli, lenticellati, fusco-corticati. Petioli ad $7.5 \mathrm{~cm}$. longi, rhachem subaequantes vel saepius ea longiores, striati, puberuli, supra haud alte canaliculati, glandula oblonga rotundatave carnosa sessili prope basem instructi; pinnae utrinsecus plerumque 3 , rarius 2 , terminales usque ad $18 \mathrm{~cm}$. longae, rhachilla glandula oblonga plana sessili inter paria duo suprema foliolorum saepius instructa; foliola utrinsecus $2-5$, oblonga vel oblongo-obovata vel terminalia obovata vel oblongo-obovata, apice rotundata, brevissime apiculata, basi plus minusve inaequilateralia, usque ad $7.5 \mathrm{~cm}$. longa et $4 \mathrm{~cm}$. lata, firme chartacea, supra glabra, nitida, subtus pilis brevibus pallide brunneis adpressis praetereaque pilis longioribus divaricatis ad costam nervosque praesertim versus costae basem instructa, nervis lateralibus utrinque ad 6 supra prominulis subtus prominentibus; stipulae fugaces. Capitula in paniculam grandem juventute ferrugineo-tomentellam disposita, pedunculo usque ad $3.5 \mathrm{~cm}$. longo striato puberulo suffulta; alabastra albo-pubescentia. Calyx $2 \mathrm{~mm}$. longus, extra aureobrunneo-puberulus, intra glaber, dentibus minutis. Corolla $5 \mathrm{~mm}$. longa, lobis oblongo-lanceolatis acutis $2 \mathrm{~mm}$. longis extra pubescentibus intra glabras. Staminum tubus irregularis, ad $4 \mathrm{~mm}$. altus, parte filamentorum libera usque ad $3 \mathrm{~cm}$. longa. Ovarium glabrum, stylo $3.5 \mathrm{~cm}$. longo. Legumen ad $19 \mathrm{~cm}$. longum et $2.2 \mathrm{~cm}$. latum, rubro-brunneum, glabrum, valvis ad semina prominenter laxe reticulatis.

Indo-China. Burma : Maymyo Plateau, 1000 m., Lace 5910.

Oxyspora rupicola, Lace [Melastomaceae-Oxysporeae]; ab O. cernua, Hook. f. et Thoms., inter alia foliis conspicue minoribus recedit.

Suffrutex sarmentosus, ramulis primo obscure quadrangulis sulcatis minute squamosis mox glabris et conspicue quadrangulis 
fistulosis pallide brunneo- vel cinereo-corticatis. Folia lanceolata, oblongo-lanceolata, apice acuminata, acuta, basi rotundata lateve cuneata, ad $13.8 \mathrm{~cm}$. longa et $4.5 \mathrm{~cm}$. lata, chartacea, pagina superiore minute tuberculata, inferiore pallidiora, glabra, e basi 5 -nervia, nervis primariis et secondariis supra conspicuis subtus prominentibus, distanter minute serrulata, petiolo ad $3.5 \mathrm{~cm}$. longo supra canaliculato summo apice barbato-piloso suffulta. Inflorescentia terminalis, pedunculo communi incluso ad $21 \mathrm{~cm}$. longa et $7 \mathrm{~cm}$. diametro, e cymis 3-7 floris sed saepissime 3-1-floris oppositis pedunculo usque ad $2 \cdot 8 \mathrm{~cm}$. longo suffultis constituta; pedicelli circa $5 \mathrm{~mm}$. longi; bracteae pedunculorum partialium $3 \mathrm{~mm}$. longae, iis pedicellorum duplo longiores. Receptaculum oblongoturbinatum, $8 \mathrm{~mm}$. longum. Calyx brevis, lobis acuminatis dorso carinatis. Petala viva punicea, obovato-elliptica, apice caudatoacuminata, $1 \cdot 1 \mathrm{~cm}$. longa, $7 \mathrm{~mm}$. lata. Antherae longiores $1 \mathrm{~cm}$. longae, breviores $6 \mathrm{~mm}$. longae, connectivo basi producto praesertim in antheris "brevioribus quarum calcar $1 \mathrm{~mm}$. longum; filamenta 6-7 mm. longa. Stylus $1.4 \mathrm{~cm}$. longus, saepe uncinatus.

Indo-China. Burma : near Maymyo Plateau; Gokteik Gorge, on rocks in middle of stream, 300 m., Lace 4978.

Lonicera Buchananii, Lace [Caprifoliaceae-Lonicereae]; a L. macrantha, DC. et L. Leschenaultii, Wall., foliis basi cuneatis nec truncatis nec cordatis indumento tenuiore subtus obtectis distinguenda.

Frutex? scandens, ramulis teretibus tomento molli griseo-albo brevi plus minusve deciduo tectis. Folia late ovata, apice acuta, mucronata, basi cuneata, ad $4.5 \mathrm{~cm}$. longa et $2.7 \mathrm{~cm}$. lata, chartaceo-membranacea, supra nitida, costa nervisque basem versus exceptis glabra, subtus glauca et pilis brevissimis albis pubescentia, nervis lateralibus utrinsecus plerumque 5 gracilibus arcuatis intra marginem integram parum recurvam anastomosantibus supra impressis subtus prominentibus; petioli graciles, supra canaliculati, 5-7 mm. longi, pubescentes. Pedunculi axillares, biflori, 1.2-2.2 $\mathrm{cm}$. longi, superne parum incrassati; bracteae binae, anguste lanceolatae, petiolatae, $0 \cdot 5-1 \cdot 4 \mathrm{~cm}$. longae, indumento ei foliorum simili instructae; bracteolae ovatae, apice rotundatae acutaeve, paulo ultra $1 \mathrm{~mm}$. longae, ciliatae. Receptaculum ovoideum, $3 \mathrm{~mm}$. altum, inferne subglabrum, superne sparse pubescens. Calycis tubus vix $1 \mathrm{~mm}$. longus, dentes 5, deltoidei, acuti, $1 \mathrm{~mm}$. longi, dorso pubescentes, intra breviter adpresse pubescentes, ciliati. Corollae tubus gracilis, superne parum ampliatus, $3 \mathrm{~cm}$. longus, extra pilis albis deflexis puberulus, intra pubescens, limbo bilabiato $2.5 \mathrm{~cm}$. longo; labium posticum 4-lobum, lobis $3-5 \mathrm{~mm}$. longis lateralibus quam medianis saepe longioribus apice obtusis margine parum undulatis, anticum circiter $2 \mathrm{~mm}$. latum. Stamina exserta, antheris curvatis circa $4 \mathrm{~mm}$. longis, filamentis glabris. Stylus filiformis, stamina superans, $6 \mathrm{~cm}$. longus, glaber, stigmate capitato.

Injo-Cnina. Burma: Myitkyina District, Watugyi, $180 \mathrm{~m}$. , E. M. Buchanan 11 (anno 1912). 
Marsdenia carnosa, Lace [Asclepiadaceae-Marsdenieae]; ab affini $M$. Jenkinsii, Hook. f., pedicellis brevioribus floribusque conspicue minoribus recedit.

Frutex volubilis, caule crasso fistuloso; ramuli teretes, striati, tomento brevi denso griseo-brunnea tecti. Folia ovata vel ovatolanceolata, basi cordata truncatave, apice subitius acuminata, obtusa, quoad magnitudinem variabilia, usque ad $15 \mathrm{~cm}$. longa et $10 \mathrm{~cm}$. lata, sicco coriacea, pagina utraque puberula, inferiore pallidora, nervis lateralibus utrinsecus $6-8$ primo obliquis rectis dein marginem versus curvatis intra marginem anastomosantibus supra prominentibus subtus saepissime valde latis planisque, nervis transversis paucis inconspicuis; petioli ad $7.5 \mathrm{~cm}$. longi, canaliculati, indumento ut caulis instructi. Inflorescentia axillaris, densa, globosa, $2-3 \mathrm{~cm}$. diametro; pedunculus communis $1.5-3 \mathrm{~cm}$. longus, ut ramuli tomentosus; pedicelli $0.5 \mathrm{~cm}$. longi, pilis brevibus pallide brunneis dense tomentosi. Calyx.5-partitus, segmentis oblongis vel obovato-oblongis apice obtusis $4 \mathrm{~mm}$. longis breviter ciliatis interioribus margine hyalinis omnibus dorso ut pedicellis tomentosis intra glabris. Corollae tubus superne dilatatus, circa $3 \mathrm{~mm}$. longus, lobis 5 dextrorsum tortis apice rotundatis $3 \mathrm{~mm}$. longis ciliolatis dorso glabris intra praesertim ad faucem villosis. Coronae squamae summo apice tantum liberae, inferne crassae, carnosae et ad staminum tubum adnatae, staminibus circiter dimidio breviores. Antherarum apices membranaceae $1 \mathrm{~mm}$. longae. Ovarium glabrum.

Indo-China. Burma: Maymyo Plateau, 1050 m., Lace 6143; English 31.

Swertia kachinensis, Lace [Gentianaceae-Swertieae]; a S. angustifolia, Ham., floribus magis numerosis, ramulis copiosis adscendentibus recedit.

$H$ erba erecta, ramulis numerosis adscendentibus, caule 4-angulato vel 4-alato rubido pallideve brunneo $40-55 \mathrm{~cm}$. alto. Folia subsessilia, lanceolata vel late lanceolata, subacuta, $4-5 \mathrm{~cm}$. longa, $1 \mathrm{~cm}$. lata, chartacea, glabra, e basi trinervia, nervis subtus prominentibus, nervis transversis subobscuris; ramorum floriferorum folia minora, anguste lanceolata. Flores numerosissima, 1-5 in axillo quoque, 4-meri ; pedicelli $0.5-1 \mathrm{~cm}$. longi. Sepala linearia, subacuta, 6-9 $\mathrm{mm}$. longa, $1 \mathrm{~mm}$. lata, trinervia. Corollae segmenta ovata, acuta, basi angustata, $7 \mathrm{~mm}$. longa, $4 \mathrm{~mm}$. lata, purpureo-maculata vel lineolata, margine incurva, glandula unica magis minusve transverse elliptica squama fimbriato-marginata late ovata quam glandula majore $1 \mathrm{~mm}$. longa obtecta basi ornata. Filamenta complanata, $3 \mathrm{~mm}$. longa, antheris oblongis. Ovarium ambitu oblongo lanceolatum.

Indo-China. Burma; Ruby Mines District; Bernardmyo, 1540 m., Lace 6307.

Utricularia brevilabris, Lace [Lentibulariaceae]; ab U. hirta, Klein, cui affinis, scapo glabro, labio inferiore quam calcare saltem dimidio breviore differt.

Herba terrestris, erecta, $2-11 \mathrm{~cm}$. alta. Folia spatulata vel lineari-spatulata, saepius $2-4 \mathrm{~mm}$. longa et paulo ultra $1 \mathrm{~mm}$, 
lata, basi in petiolum plerumque lamina longiorem angustata. Scapus gracillimus, simplex, glaber, flores usque ad 5 gerens, squamis paucis lanceolatis longe acuminatis basi affixis circa $1 \mathrm{~mm}$. longis instructus; pedicelli $1-2 \mathrm{~mm}$. longi; bracteae squamis similes; bracteolae binae, minutissimae. Calycis segmenta inter se inaequalia, obovata, apice rotundata, circa $1.5 \mathrm{~mm}$. longa, inferiore quam superiore latiore et paulo breviore. Corolla purpurea, ore minutissime puberula; labium posticum oblongooblanceolatum, apice obtusum, $2.5 \mathrm{~mm}$. longum, $1 \mathrm{~mm}$. latum, anticum trilobatum, lobis rotundatis, vivum 4-5 mm. latum, circa $2 \mathrm{~mm}$. longum, maculis duobus luteis basi ornatum; calcar $5 \mathrm{~mm}$. longum, gracile, apicem versus subacutum angustatum, horizontale, rectum vel apice parum sursum curvatum. Semina laxe et graciliter reticulata.

Indo-China. Burma: Maymyo Plateau, in swampy ground, 1050 m., Lace 5489..

Uricularia Rogersiana, Lace [Lentibulariaceae]; ab affini $U$. punctata, Wall., partibus omnibus multo gracilioribus et corollae colore diverso differt.

Herba natans. Folia submersa, multipinnata, segmentis ultimis capillaribus utriculis numerosis instructis. Scapus erectus, gracilis, simplex, sparse puberulus, 2-4-florus; squamae suborbiculares, apice rotundatae, basi cordata, bracteis minores; pedicelli 3-6 $\mathrm{mm}$. longi; bracteae late ovatae, apice obtusae, basi cordatae, $2 \mathrm{~mm}$. latae, vix $2 \mathrm{~mm}$. longae. Calycis segmenta suborbicularia, apice rotundata, emarginulata, superiore ultra $2 \mathrm{~mm}$. longo et lato quam inferiore paulo majore. Corolla pallida, purpureo-venosa; labium superum oblongo-ovatum, apice obtusum, $5 \mathrm{~mm}$. longum, inferum suborbiculare, apice rotundatum, $8 \mathrm{~mm}$. longum, $7 \mathrm{~mm}$. latum; calcar circa $1.75 \mathrm{~mm}$. longum, superne subito breviter angustatum, summo apice obtusum. Ovarium ovoideum, paulo ultra $1 \mathrm{~mm}$. altum, glabrum.

Indo-China. Burma: Maymyo Plateau, 1050 m., Lace 5899. Named after Mr. C. Gilbert Rogers, Conservator of Forests, who kindly assisted in the collection of this plant and many others.

Utricularia subrecta, Lace [Lentibulariaceae]; ab U. caerulea, Linn., caule graciliore, floribus majoribus, calcare longiore acuto recedit.

Herba erecta interdum volubilis 6-30 cm. alta, glabra. Folia linearia vel lineari-spatulata, 1-3 cm. longa, vix ultra $1.5 \mathrm{~mm}$. lata, apice rotundata vel obtusa, basi in petiolum usque ad $3 \mathrm{~cm}$. longum angustata; utriculi breviter petiolati $1.5 \mathrm{~mm}$. diametro. Scapus simplex, flores 1-7 (saepissime 3-5) gerens, squamis paucis acutis $2-3 \mathrm{~mm}$. longis basi haud productis; pedicelli ad $1 \mathrm{~cm}$. longi; bracteae persistentes, squamis similes; bracteolae binae, anguste lineares, circa $2 \mathrm{~mm}$. longae. Calycis segmenta inter se subaequalia, ovata, acuminata, acuta, 4-5 mm. longa. Corolla caerulea vel puniceo-purpurea ; labium superum lineari-oblongum, emarginatum, 6-7 mm. longum, $2 \mathrm{~mm}$. latum, infimum lateribus reflexum, vivum $5 \mathrm{~mm}$. diametro, emarginatum, ore pilis brevibus instructum; calcar $6-8 \mathrm{~mm}$. longum, anguste conicum, apice acutum, parum arcuatum vel sicco fere rectum, angulum 
rectum cum labio infimo efficiens. Capsula ovoidea, in calyce inclusa; semina globosa, longitudinaliter brunneo-costata.$U$. caerulea, Coll. et Hemsl. in Jouru. Linn. Soc., vol. xxviii, p. 101, vix Linn.

Indo-China. Burma: Shan Hills Plateau; Koni, 1540 m., Collett 469; Maymyo Plateau, 1050 m., Lace 3151, 4845, 5488, 5505.

Strobilanthes mogokensis, Lace [Acanthaceae-Ruellieae]; a S. tamburense, C. B. Clarke, spicis angustioribus, bracteis lanceolato-oblongis, corolla longiore recedit.

Frutex lignosus, caulibus sulcatis pallide brunneis praesertim ad nodos lenticellatis; ramuli juventute pilis albis glandulosis transverse septatis tecti, demum glabri. Folia late ovata, breviter acuminata, summo apice obtusa, inferiora basi truncata vel subito anguste cuneata, superiora truncata, sessilia, anguste cordata, usque ad $6 \mathrm{~cm}$. longa, $4.5 \mathrm{~cm}$. lata, chartacea pilis albis latis transverse septatis pagina utraque glanduloso-pubescentia, nervis lateralibus utrinsecus 6-7 tenuibus supra conspicuis subtus prominulentibus, margine crenulata, ciliata; petioli foliorum inferiorum ad $1.5 \mathrm{~cm}$. longi, supra canaliculati. Spicae et ramos et ramulos breves laterales terminantes et pauce in foliis supremis axillares, glanduloso-pubescentes, ad $5 \mathrm{~cm}$. longae, vix $1 \mathrm{~cm}$. diametro; bracteae lanceolato-oblongae, $1 \mathrm{~cm}$. longae; bracteolae anguste lanceolatae, 7-8 $\mathrm{mm}$. longae, ut bracteae extra glandulosopubescentes, intra glabrae. Calyx $1 \mathrm{~cm}$. longus, fere ad basem bilabiatus, labio altero magis minusve ad medium lobato altero fere ad basem 2-fido, lobis omnibus lanceolatis acute acuminatis ciliatis dorso glanduloso-pubescentibus intra adpresse pubescentibus. Corolla arcuata, praeclare late ventricosa, $3 \mathrm{~cm}$. longa, extra sparse pubescens intra nisi ad staminum insertionem glabra, parte cylindrica $8 \mathrm{~mm}$. longa, lobis rotundatis. Stamina 4, filamentis fere glabris nisi ad basem. Ovarium pilis erectis albobrunneis apice pennicellatum, stylo $2.5 \mathrm{~cm}$. longo superne pilis longiusculis albis divaricatis instructo. Capsula $1 \mathrm{~cm}$. longa, apicem versus pubescens; semina $2.5 \mathrm{~mm}$. longa, fere $2 \mathrm{~mm}$. lata, complanata, pilis brevibus elasticis tecta.

Indo-China. Burma: Ruby Mines District, Bernardmyo to Mogok, 1850 m., Lace 6018.

Gutzlaffia glandulosa, Lace [Acanthaceae-Ruellieae]; habitu $G$. exareolatae,* similis sed seminibus haud glabris differt.

Frutex, caule sulcato fere glabro. Folia ovato-lanceolata vel ovata apice caudato-acuminata, apiculata, basi acuminata, ad $15 \mathrm{~cm}$. longa, $7 \mathrm{~cm}$. lata, chartaceo-membranacea, pagina superiore pilis albis crassis hic illic instructa, inferiore ad nervos pilis adpressis reflexis dense instructa praetereaque pilis albis crassis tranverse septatis ad nervos nervulosque praesertim superne et marginem versus additis, nervis lateralibus utrinsecus 8-10 arcuatis supra conspicuis subtus prominentibus, acumine excepto serrato-crenata, petiolo $0.5-2.5 \mathrm{~cm}$. longo breviter pubescente

* Gutzlaffia exareolata, Lace, comb. nov. = Strobilanthes exareolatus, C. B. Clarke. 
supra canaliculato suffulta. Spicae glanduloso-pilosae, laxae, angustae, usque ad $6 \mathrm{~cm}$. longae, in paniculas grandes terminales dispositae; flores oppositi, alabastris glanduloso-pubescentibus; bracteae $3 \mathrm{~mm}$. longae, late truncatae, apice parum retusae; bracteolae lineares, apice rotundatae. Calyx subaequaliter 5-fidus, $5 \mathrm{~mm}$. longus, segmentis anguste linearibus vix $1 \mathrm{~mm}$.latis ciliatis. Corolla alba, purpureo-suffusa, $3 \mathrm{~cm}$. longa, ore $1 \mathrm{~cm}$. diametro, inferne gradatim angustata, lobis rotundatis extra superne sparse pubescentibus intra glabris nisi lobi inferioris apicem versus. Stamina 2, filamentis complanatis glabris. Ovarium $7 \mathrm{~mm}$. altum, pilis albis erectis dense coronatum. Semina ultra $2 \mathrm{~mm}$. longa, $1.5 \mathrm{~mm}$. lata, basi excepta pilis brevibus elasticis tecta.

Indo-China. Burma: Ruby Mines District; Bernardmyo to Mogok, 1850 m., Lace 6019.

\title{
XLII.-MIMOSA CAESIA AND M. INTSIA.
}

\author{
W. G. CRAIB.
}

The somewhat divergent views adopted with regard to these two Linnean species at different times by successive authors prompted the writer to an attempt to clear up the mystery surrounding them.

With $M$. caesia there seems to be practically no difficulty. Linnaeus based his species on Fl. Zeyl. 217, and of this there exist three specimens in Herb. Mus. Brit. which agree among themselves and which are identical with Acacia Intsia, Wight et Arn. In addition to the Fl. Zeyl. Linnaeus also quotes Plukenet. Unfortuately there does not appear to exist a specimen of Plukenet's plant, but from the figure the writer feels disposed to refer it, not to the Fl. Zeylanica plant but to the plant enumerated below as Acacia torta. According to Jackson there is a specimen written up as caesia in the Linnean herbarium, but not in Linnaeus's own handwriting. The writer proposes to limit M. caesia to Hermann's plant which is unquestionably the plant intended by Linnaeus.

Exactly which plant Linnaeus meant by his $M$. Intsia the writer has found impossible to decide. The first plant quoted by Linnaeus in his Sp. Pl., i. p. 522 is that of Hort. Cliff. 209. No trace of this particular Cliffortian plant was found in Herb. Mus. Brit., but a reference to the description reveals the fact that at least two plants are here involved. Linnaeus says concerning his plant " crescit in Madera et Malabaria et variis Americae partibus, unde semina omni anno nobis allata fuere." From this word sequence one might be justified in assuming that the Cliffortian plant was grown not from Indian but from American seed. If this were so, and since no species of this group of Acacia is common to India and America, we have here sufficient grounds for excluding the species from the Indian flora.

Further, Linnaeus in both the Hort. Cliff. and Sp. Pl. quotes references to two Indian plants, viz., Pluk. Alm., iv. t. 122 f. 2 and Rheede, Hort. Mal., vi. t. 4. The former figure shows what is, judging from the pods, an Acacia, but an examination of the 